\title{
Imaging Charge Localization in a Conjugated Oligophenylene
}

\author{
Laerte L. Patera $\odot$, Fabian Queck, and Jascha Repp® \\ Institute of Experimental and Applied Physics, University of Regensburg, 93053 Regensburg, Germany
}

(Received 19 May 2020; revised 23 July 2020; accepted 8 September 2020; published 23 October 2020)

\begin{abstract}
Polaron formation in conjugated polymers has a major impact on their optical and electronic properties. In polyphenylene, the molecular conformation is determined by a delicate interplay between electron delocalization and steric effects. Injection of excess charges is expected to increase the degree of conjugation, leading to structural distortions of the chain. Here we investigated at the single-molecule level the role of an excess charge in an individual oligophenylene deposited on sodium chloride films. By combining sub-molecular-resolved atomic force microscopy with redox-state-selective orbital imaging, we characterize both structural and electronical changes occurring upon hole injection. While the neutral molecule exhibits a delocalized frontier orbital, for the cationic radical the excess charge is observed to localize, inducing a partial planarization of the molecule. These results provide direct evidence for selftrapping of the excess charge in oligophenylenes, shedding light on the interplay of charge localization and structural distortion.
\end{abstract}

DOI: 10.1103/PhysRevLett.125.176803

Excess charges in dielectric solids tend to localize as small polarons, due to self-induced potentials resulting from strong carrier-lattice interactions [1-5]. In conjugated polymers, excess charges induce local distortions of the molecular structure [6] and the appearance of polaronic states in the band gap [7]. The formation of in-gap states strongly affects the optical and the electronic properties of conjugated polymers $[6,8]$. In poly ( $p$-phenylene), despite conjugation favors a planar configuration by maximizing the orbital overlap between adjacent $p_{z}$ orbitals, steric effects arising from repulsion between the orthohydrogens induce a twist away from the planar conformation. Once a charge is injected, the torsional angle between adjacent phenyl rings is reduced, reflecting an energy gain upon orbital delocalization [6,9-11]. Because of the delicate interplay between conjugation and structural distortion, this class of nonrigid compounds provides a convenient platform for examining charge localization in conjugated polymers. However, the degree of polaron extension predicted by theoretical calculations strongly depends on the approach [12-14]. While pure density functional theory (DFT) functionals overestimate the charge delocalization [15], hybrid DFT [16], and semiempirical Hartree-Fock [17] methods yield the formation of well-defined polarons. Further, the accuracy of the different approaches in predicting the polaronic character of the charge carriers seems to depend on the type of system under study [9]. Moreover, despite that electron nuclear double resonance spectroscopy $[18,19]$ has been used to study charge delocalization in thin films of conjugated polymers, a direct experimental access to the spatial extension of polaronic states in individual oligophenylenes has remained out of reach. In particular, the presence of a polarizable substrate, or polar solvents, is expected to play a role in further determining the degree of charge localization [20]. Here, we studied the effect of hole injection on the structure and electronic properties of an individual oligophenylene, namely, parasexiphenyl $\left(\mathrm{C}_{36} \mathrm{H}_{26}, 6 P\right)$, deposited on $\mathrm{NaCl}$ films. While noncontact atomic force microscopy (AFM) allows resolving the molecular adsorption geometry and mapping the excess charge distribution, redox-state selective orbital imaging provides direct evidence of orbital localization.

Experiments were carried out with a low-temperature scanning tunneling and atomic force microscope (STM/ AFM) equipped with a qPlus tuning fork [21] (resonance frequency $f_{0} \approx 29.1 \mathrm{kHz}$, spring constant $k=1.8 \mathrm{kNm}^{-1}$, quality factor $Q \approx 3 \times 10^{4}$ ) in ultrahigh vacuum $\left(p \approx 2 \times 10^{-10}\right.$ mbar) and at a temperature of $7 \mathrm{~K}$. As substrates we used a $\mathrm{Cu}(111)$ single crystal covered with different thicknesses of $\mathrm{NaCl}$. The molecules were deposited onto the sample kept at $\approx 8 \mathrm{~K}$ inside the microscope. Bias voltages are given as sample bias with respect to the tip. Positive constant-height offsets $\Delta z$ correspond to a distance increase with respect to the STM/AFM set point above the $\mathrm{NaCl}$. For single-electron alternate charging scanning tunneling microscopy (AC-STM) experiments [22], ac voltage $\left(V_{\mathrm{ac}}\right)$ pulses were produced by an arbitrary waveform generator (Agilent 33522A) and fed to the microscope head through semirigid coaxial high-frequency cables. $V_{\mathrm{ac}}$ values are given in volts peak to peak $\left(\mathrm{V}_{\mathrm{pp}}\right)$. Orbital calculations were performed using the ORCA (4.0.1) program [24]. The B3LYP density functional, in combination with the correlation-consistent double-zeta (cc-pVDZ) basis set, was used.

The chemical structures of the neutral $\left(6 P^{0}\right)$ and cationic $\left(6 P^{+}\right)$molecules are shown in Fig. 1(a). In the cationic 
(a)
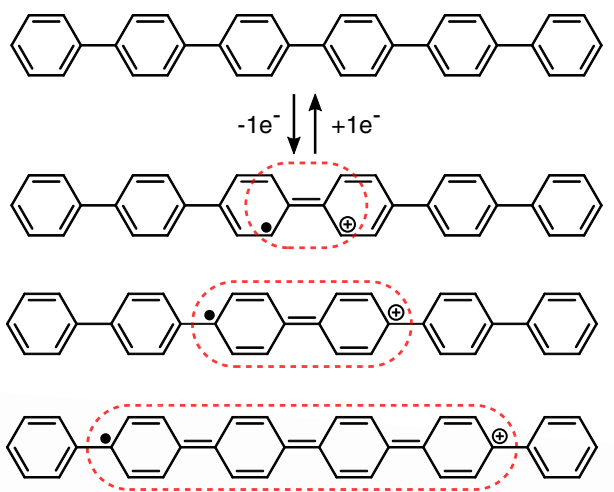

(b)

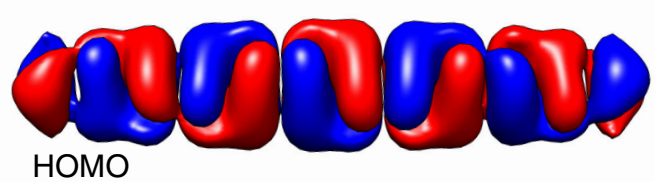

FIG. 1. (a) Chemical structures of neutral (top) and three resonance structures for the cationic species of $6 P$. For the cationic radical, a transition to a quinoid structure is directly linked to the separation of hole and unpaired electron, as is highlighted in red. (b) Calculated HOMO contour of gas-phase $6 P^{0}$.

radical, the positive charge induces a local transformation of the structure [9], from benzenoid to quinoid. However, since the delicate balance between conjugation and structural distortion is not captured in the Lewis resonance structures, the actual extension of the charge distribution cannot be easily predicted. As a result, while the highest occupied molecular orbital (HOMO) of the neutral gasphase molecule [Fig. 1(b)] is expected to be delocalized over the entire molecule, upon hole injection the spatial distribution of this orbital, becoming singly occupied, is a priori not known.

We first investigated the adsorption geometry of individual $6 P$ molecules once deposited on a $\mathrm{NaCl}$ surface. Adsorbed on a two monolayer (ML) thick $\mathrm{NaCl}$, the molecules maintain the neutral charge state, as indicated by the absence of scattering of the interface state in scanning tunneling microscopy (STM) images [25] (see Supplemental Material [26]). AFM with COfunctionalized tips $[23,27,28]$ has been employed to characterize the adsorption configuration of the $6 P^{0}$ on $\mathrm{NaCl}(2 \mathrm{ML}) / \mathrm{Cu}(111)$. Figure 2(a) shows a map of the cantilever's frequency shift $(\Delta f)$ acquired at a relatively large tip-sample distance. The contrast is dominated by features at negative $\Delta f$ values, corresponding to attractive van der Waals (vdW) interactions. Despite the longrange nature of these interactions, the image exhibits submolecular features, suggesting a nonplanar adsorption configuration. This is in line with previous studies of conjugated oligophenylenes deposited on weakly interacting substrates [29-32]. At closer distances [Fig. 2(b)], less attractive features emerge, corresponding to bonds that emerge further towards the tip than other parts of the molecule [33-35]. Because of the low temperatures and the adsorption on the $\mathrm{NaCl}$ surface, the phenyl rings cannot freely rotate against each other such that $6 P$ exhibits a defined axial chirality and a racemic mixture of two enantiomers is expected on the surface. Despite the nonplanarity of $6 P$, which prevents resolving the entire molecular geometry by constant-height AFM imaging, the vertical imaging method allows extracting information about the three-dimensional structure $[33,34,36]$. This approach, based on force-distance spectroscopy, enables the acquisition of data at closer tip sample distances $z$ than in constant-height imaging and therefore provides information about lower-lying structural features. We exploited this approach to estimate the value of the torsional angle $\theta$ [Fig. 2(c)]. A vertical image [Fig. 2(d)] has been acquired along the $\mathrm{C}-\mathrm{C}$ bonds parallel to the long molecular axis [see direction indicated in the inset of Fig. 2(e)]. Figure 2(e) shows the frequency shift $\Delta f$ as a function of tip height $z$ acquired above the center of the C-C bonds of the tilted phenyl rings. By determining the tip height $z^{*}$, at which $\Delta f(z)$ is minimal, it is possible to estimate the height difference between the $\mathrm{C}-\mathrm{C}$ bonds lying closer to the substrate and the ones pointing out toward the tip. A value of $\Delta z^{*} \approx 0.7 \AA$ is obtained [Fig. 2(e)]. Given the distance between two parallel $\mathrm{C}-\mathrm{C}$ bonds in a phenyl ring being $2.4 \AA, \theta$ can be estimated to be $\approx 34^{\circ}$. This is in rough agreement with the value of $40^{\circ}$, determined for gas-phase $6 P^{0}$ by semiempirical Hartree-Fock calculations [9]. Because of the absence of dipoles along the $6 P$, no influence of the junction's electric field is expected on the molecular structure. Instead, a decrease of torsional angle is likely, due the attractive adsorption potential, which tends to flatten the molecule, being partially compensated by the tip's attractive force. In addition, the experimental procedure is expected to slightly overestimate $\theta$. In fact, at closer tip-sample distances, a larger attractive background force may shift the $z^{*}$ value towards lower values, exaggerating the apparent $\theta$ value.

To study the effect of an excess charge on individual $6 P$, the molecules have been deposited on $\mathrm{NaCl}$ films exceeding 20 monolayers, which enable charge-state control [37]. Figure 3(a) shows a $\Delta f$ spectrum as a function of the dc bias $\left(V_{\mathrm{dc}}\right)$ acquired above an isolated $6 P$ molecule. A sudden change in $\Delta f(V)$ at $V_{\mathrm{dc}} \approx-3.4 \mathrm{~V}$ indicates a charge-state transition [37,38], corresponding to the first reversible oxidation of $6 P$. Figure 3(b) shows a constantheight AFM $\Delta f$ image acquired in the cationic state close to this transition $\left(V_{\mathrm{dc}}=-3.45 \mathrm{~V}\right)$. While in most of the image the molecule is positively charged $\left(6 P^{+}\right)$, spikes are present in the center (highlighted by an arrow), resulting from stochastic tunneling events [38]. These features indicate that discharging occurs preferentially when the tip is roughly above the two inner benzene rings [Fig. 4(c)]. 

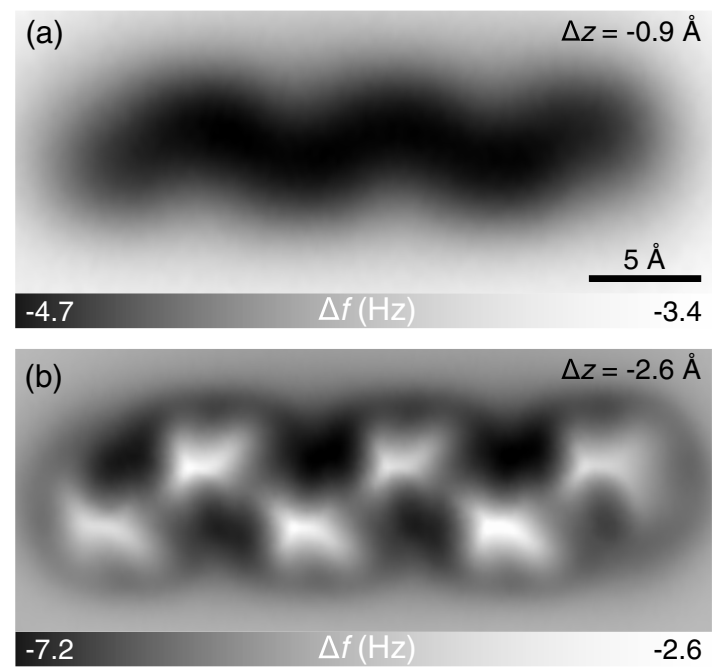

(c)

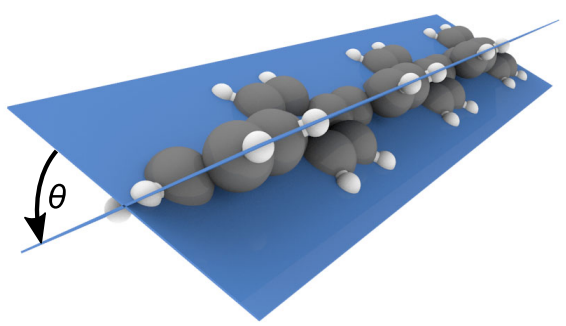

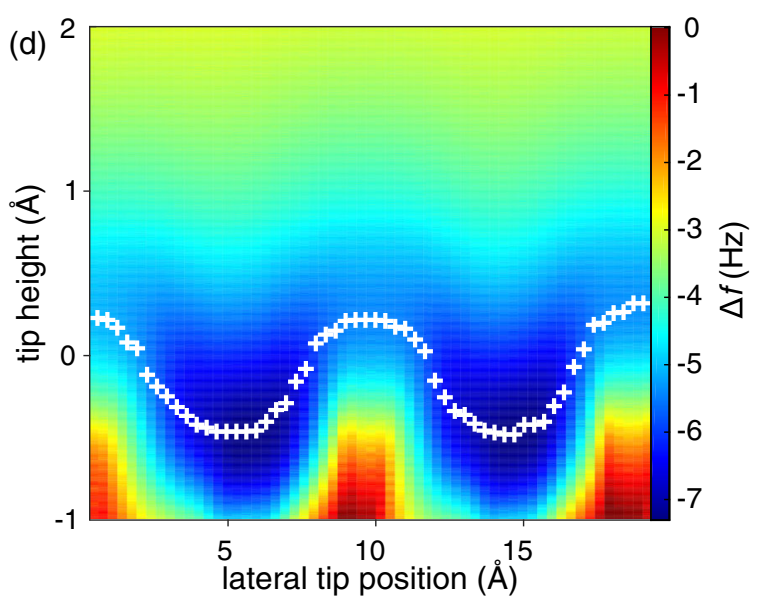

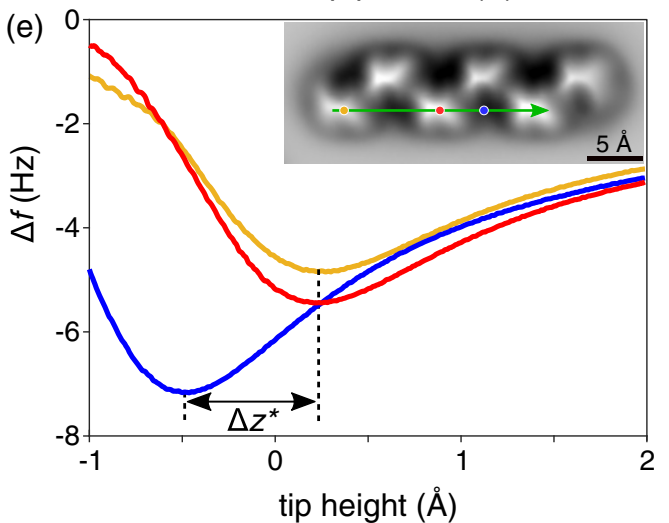

FIG. 2. (a),(b) Constant-height AFM $\Delta f$ images of $6 P^{0}$ on $\mathrm{NaCl}(2 \mathrm{ML}) / \mathrm{Cu}(111)$, acquired with a CO-functionalized tip at $\Delta z=-0.9$ (a) and $\Delta z=-2.6 \AA$ (b). $\Delta z$ is given with respect to opening the feedback loop at an STM set point of $I=0.6 \mathrm{pA}, V=0.12 \mathrm{~V}$ above the clean $\mathrm{NaCl}$ surface. AFM oscillation amplitude, $A=1 \AA$. (c) Model of gas-phase $6 P$, where the torsional angle $(\theta)$ is indicated. Gray and white spheres represent carbon and hydrogen atoms, respectively. (d),(e) Determination of the torsional angle for $6 P^{0}$. (d) Vertical image of $6 P^{0}$ acquired with a CO-functionalized tip along the direction indicated in the inset of (e). The white crosses in (d) mark the minimum $\left(z^{*}\right)$ of each $\Delta f(z)$ spectrum. (e) $\Delta f(z)$ spectra acquired above the tilted phenyl rings. Inset: an arrow indicates the direction of the vertical image, superimposed to the panel (b). (d),(e) The feedback was opened at an STM set point of $I=1.5$ pA, $V=0.30 \mathrm{~V}$. A background $\Delta f(z)$ spectrum acquired on the bare $\mathrm{NaCl}$ surface has been subtracted from all spectra in (d),(e).

This provides a first indication that the hole localizes at the center of the molecule.

To further investigate this behavior, we imaged the molecule in both the neutral and the cationic states. Figures 4(a)-4(b) show constant-height AFM $\Delta f$ images acquired with a metal tip apex. By choosing a $V_{\text {dc }}$ value below the charging threshold $\left(V_{\mathrm{dc}}=-3.20 \mathrm{~V}\right)$, the $\Delta f$ map in Fig. 4(a) corresponds to the neutral molecule. The contrast closely matches the one obtained with a $\mathrm{CO}$ tip on the thin $\mathrm{NaCl}$ films [Fig. 2(a)]. Upon hole injection $\left(V_{\mathrm{dc}}=-3.55 \mathrm{~V}\right)$, a less attractive contrast is detected at the center of the molecule [Fig. 4(b)]. This feature extends for $\approx 10 \AA$ along the main molecular axis, roughly matching the length of two adjacent phenyl rings. To interpret such change in the contrast, one has to consider the different force contributions which determine the AFM signal. In the $\Delta f$ maps in Figs. 4(a)-4(b), which have been acquired at rather large tip-sample distances, the $\Delta f$ contrast is dominated by a sum of $\mathrm{vdW}$ and electrostatic contributions $[23,39,40]$. Since for metallic tips at these distances [Figs. 4(a)-4(b)], the distance dependence of $\Delta f$ is strictly monotonic, the less attractive $\Delta f$ feature in the center of the molecule could indicate an increase in the tip-molecule distance, pointing towards a (partial) planarization of the inner benzene rings. However, as shown in Fig. 3(a), charging leads as well to a less attractive $\Delta f$ signal [40], possibly indicating charge localization towards the center. For this reason, disentangling structural relaxations from the excess charge distribution in AFM images is not straightforward.

To determine the spatial extension of the charge distribution, we performed AC-STM, which allows chargestate controlled orbital imaging of single molecules [22]. The AC-STM contrast can be directly related to the density distribution of the molecular orbitals [41], providing direct information on the spatial extension of the excess charge [22]. Figure 4(c) shows a map of the $0 \rightarrow 1^{+}$transition, corresponding to the HOMO distribution of $6 P^{0}$. The 

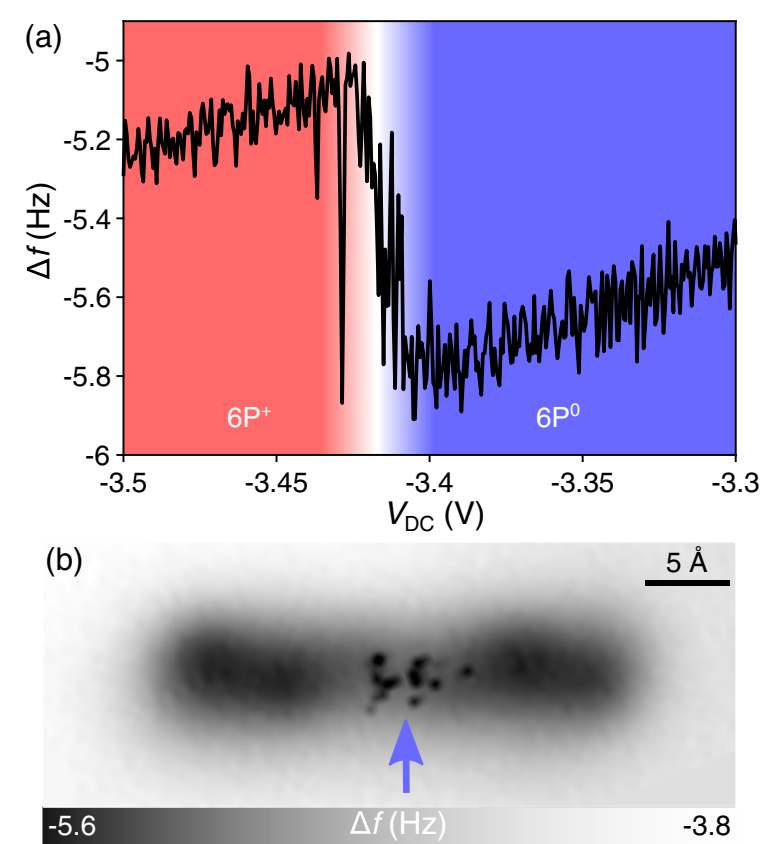

FIG. 3. Charge-state manipulation of $6 P$ on $\mathrm{NaCl}(\geq 20 \mathrm{ML}) / \mathrm{Cu}(111)$. (a) $\Delta f\left(V_{\mathrm{dc}}\right)$ spectrum acquired on an isolated molecule $(A=1 \AA, \Delta z=-2.5 \AA$ ). (b) Constantheight AFM $\Delta f$ image acquired with a metal tip apex $\left(V_{\mathrm{dc}}=-3.45 \mathrm{~V}, V_{\mathrm{ac}}=0 \mathrm{~V}, A=1 \AA, \Delta z=-2.7 \AA\right) . \Delta z$ is given with respect to opening the feedback loop at an AFM set point of $\Delta f=1 \mathrm{~Hz}, V_{\mathrm{dc}}=0 \mathrm{~V}$ above the clean $\mathrm{NaCl}$ surface.

image indicates a delocalized orbital, exhibiting two pronounced features at the termini, in agreement with the simulated image (Supplemental Material [26]). Upon probing the inverse charge-state transition $\left(1^{+} \rightarrow 0\right)$, the contrast appears to be much more localized towards the center of the molecule [Fig. 4(d)]. Notably, the change in the contrast observed in the AFM $\Delta f$ image upon charging exhibits a similar degree of localization as in the AC-STM image [Fig. 4(e)]. This observation suggests that the change in the contrast of the $\Delta f$ channel is related to the spatial distribution of the excess charge [37,42]. However, structural distortions are likely to occur due to the delicate interplay between geometry relaxation and electronic structure in oligophenylenes [8,9]. Charge screening in the $\mathrm{NaCl}$ gives rise to further stabilization [39], possibly inducing structural relaxations in the molecule $[43,44]$. Since the excess charge in the 6P is likely to be screened within the first few layers of $\mathrm{NaCl}$ [38], no strong polarization at the $\mathrm{NaCl}$-metal interface is expected. In the AFM difference image (see Supplemental Material [26]), a less-attractive contrast can be seen in the center. Since features are mostly located at the protruding sides of tilted benzene rings, rather than resembling the orbital image [Fig. 4(d)], the $\Delta f$ signal in the difference image indicates an inhomogeneous distribution of the torsional angle in the $6 P^{+}$. However, despite that AFM with
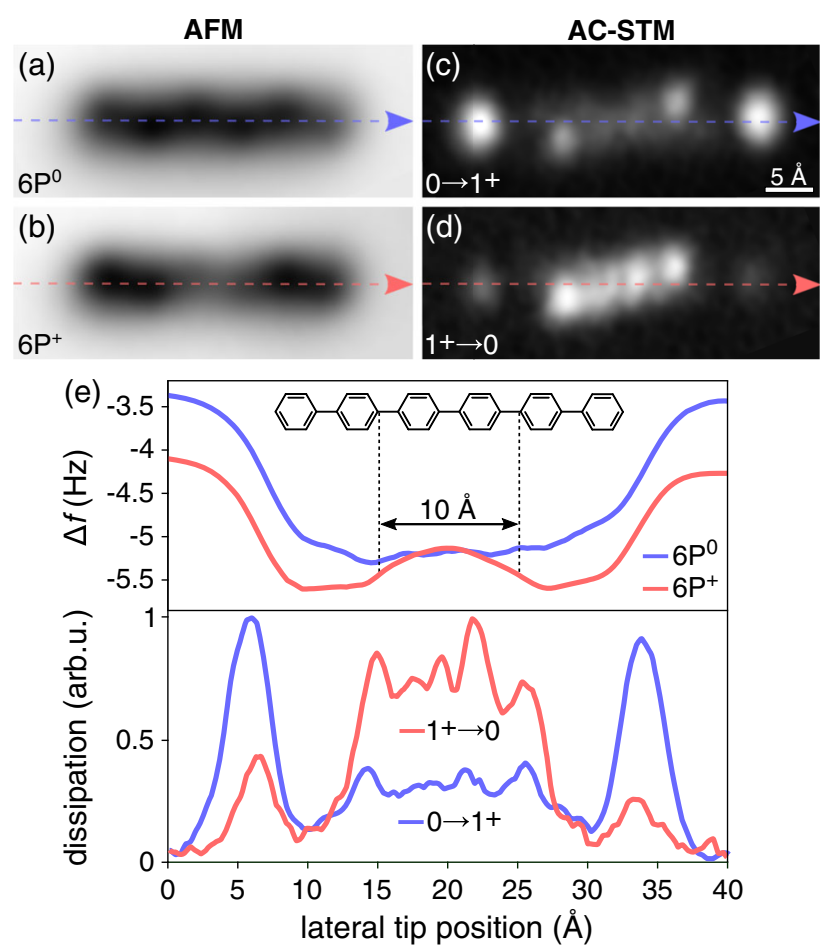

FIG. 4. Charge-state controlled imaging of $6 P$ on $\operatorname{NaCl}(\geq 20 \mathrm{ML}) / \mathrm{Cu}(111)$. (a),(b) Constant-height AFM $\Delta f$ images: $6 P^{0}$ [(a) $\left.V_{\mathrm{dc}}=-3.20 \mathrm{~V}, V_{\mathrm{ac}}=0 \mathrm{~V}_{\mathrm{pp}}, \Delta z=-2.7 \AA\right]$ and $6 P^{+}\left[\right.$(b) $\left.V_{\mathrm{dc}}=-3.55 \mathrm{~V}, V_{\mathrm{ac}}=0 \mathrm{~V}_{\mathrm{pp}}, \Delta z=-2.7 \AA\right]$. (c), (d) Constant-height AC-STM images: $0 \rightarrow 1^{+} \quad[$ (c) $\left.V_{\mathrm{dc}}=-3.25 \mathrm{~V}, V_{\mathrm{ac}}=1.40 \mathrm{~V}_{\mathrm{pp}}, \Delta z=-2.5 \AA\right] ; 1^{+} \rightarrow 0[(\mathrm{~d})$ $\left.V_{\mathrm{dc}}=-3.32 \mathrm{~V}, V_{\mathrm{ac}}=1.40 \mathrm{~V}_{\mathrm{pp}}, \Delta z=-2.7 \AA\right]$. All the images have been acquired with the same metal tip apex and $A=1 \AA$. $\Delta z$ is given with respect to opening the feedback loop at an AFM set point of $\Delta f=-1 \mathrm{~Hz}, V_{\mathrm{dc}}=0 \mathrm{~V}$ above the clean $\mathrm{NaCl}$ surface. (e) Line profiles of AFM (top) and AC-STM (bottom) images.

CO-functionalized tips has been recently exploited to precisely detect conformation changes [44], the inhomogeneous spatial distribution of the excess charge poses a challenge to disentangle structural contributions from electrostatic ones, hindering a quantitative determination of the structural changes [22].

Now we turn the discussion to the extension of the polaronic state. The orbital localization observed for the $6 P^{+}$[Fig. 4(d)] may—at first glance-come as a surprise, because of the expected increase in the degree of overall conjugation upon charging and a stronger conjugation being often accompanied by a more delocalized orbital distribution. However, for such kind of conjugated polymers, due to the delicate interplay of conjugation and torsional angle, conjugation and delocalization do not generally coincide. From tight-binding band-structure considerations, as long as all benzene rings feature an equivalent geometry, the frontier orbitals display equal weight along the phenyl chain (disregarding end-state effects). Localization, instead, requires removing the 
equivalence of the phenyl units (in terms of mutual overlap integrals), which is the case for $6 P^{+}$having different torsional angles. Gas-phase semiempirical Hartree-Fock calculations predicted a partial planarization of the $6 \mathrm{P}^{+}$[9]. This is in good agreement with the observed AFM image contrast [Figs. 4(a)-4(b)] (see Supplemental Material [26]). Nevertheless, in our experiments the possible role of the dielectric environment has also to be considered. In fact, the formation of a polaron at the molecule- $\mathrm{NaCl}$ interface can cause a localization of the electronic wave functions [22]. While the HOMO of $6 \mathrm{P}^{0}$ extends for $\approx 30 \AA$ [Fig. 4(c)$4(\mathrm{e})]$, for the $6 \mathrm{P}^{+}$the excess charge localizes in a region of $\approx 13 \AA$ (see Supplemental Material [26]), in agreement with the polaron extension predicted by semiempirical HartreeFock calculations of gas-phase molecules [9]. This may indicate that the charge localization given by the strong intramolecular relaxations dominates over the contribution given by the polaron formation in the underlying ionic substrate.

In conclusion, by combining sub-molecular-resolved AFM with charge-state-selective orbital imaging, we studied structural and electronic changes occurring upon hole injection in a prototypical oligophenylene deposited on $\mathrm{NaCl}$ films. While the neutral molecule exhibits a delocalized frontier orbital and a homogeneous distribution of torsional angles between the adjacent benzene rings, for the cationic radical the excess charge is observed to localize towards the center, inducing a partial planarization of the molecule. These results provide direct evidence for orbital localization upon polaron formation in conjugated oligophenylenes, paving the way to the study of the distribution of excess charges in extended conductive polymers.

We thank Philipp Scheuerer and Ferdinand Evers for discussion. Funding from Deutsche Forschungsgemeinschaft (DFG, German Research Foundation), Project IDs RE2669/6 and 314695032 SFB 1277 , is gratefully acknowledged.

*laerte.patera@physik.uni-regensburg.de

[1] A. Shluger and A. Stoneham, J. Phys. Condens. Matter 5, 3049 (1993).

[2] T. Holstein, Ann. Phys. (Leipzig) 8, 325 (1959).

[3] M. Setvin, C. Franchini, X. Hao, M. Schmid, A. Janotti, M. Kaltak, C. G. Van de Walle, G. Kresse, and U. Diebold, Phys. Rev. Lett. 113, 086402 (2014).

[4] I. Austin and N. F. Mott, Adv. Phys. 18, 41 (1969).

[5] C. M. Yim, M. B. Watkins, M. J. Wolf, C. L. Pang, K. Hermansson, and G. Thornton, Phys. Rev. Lett. 117, 116402 (2016).

[6] J. L. Bredas and G. B. Street, Acc. Chem. Res. 18, 309 (1985).

[7] N.-H. Ge, C. Wong, R. Lingle, J. McNeill, K. Gaffney, and C. Harris, Science 279, 202 (1998).

[8] J. Bredas, B. Themans, J. Andre, R. Chance, and R. Silbey, Synth. Met. 9, 265 (1984)
[9] E. Zojer, J. Cornil, G. Leising, and J. L. Brédas, Phys. Rev. B 59, 7957 (1999).

[10] S. Arulmozhiraja and T. Fujii, J. Chem. Phys. 115, 10589 (2001).

[11] A. Ye, Z. Shuai, O. Kwon, J.-L. Brédas, and D. Beljonne, J. Chem. Phys. 121, 5567 (2004).

[12] I. H. Nayyar, E. R. Batista, S. Tretiak, A. Saxena, D. Smith, and R. L. Martin, J. Phys. Chem. Lett. 2, 566 (2011).

[13] A. J. Cohen, P. Mori-Sánchez, and W. Yang, Science 321, 792 (2008).

[14] M.-C. Kim, E. Sim, and K. Burke, Phys. Rev. Lett. 111, 073003 (2013).

[15] L. Zuppiroli, A. Bieber, D. Michoud, G. Galli, F. Gygi, M. Bussac, and J. André, Chem. Phys. Lett. 374, 7 (2003).

[16] V. Geskin, A. Dkhissi, and J. Brédas, Int. J. Quantum Chem. 91, 350 (2003).

[17] G. Moro, G. Scalmani, and U. Cosentino, and D. Pitea, Synth. Met. 108, 165 (2000).

[18] S. Kuroda, K. Marumoto, Y. Shimoi, and S. Abe, Thin Solid Films 393, 304 (2001).

[19] R. Steyrleuthner, Y. Zhang, L. Zhang, F. Kraffert, B. P. Cherniawski, R. Bittl, A. L. Briseno, J.-L. Bredas, and J. Behrends, Phys. Chem. Chem. Phys. 19, 3627 (2017).

[20] A. T. Yeh, C. V. Shank, and J. K. McCusker, Science 289, 935 (2000).

[21] F. J. Giessibl, Appl. Phys. Lett. 73, 3956 (1998).

[22] L. L. Patera, F. Queck, P. Scheuerer, and J. Repp, Nature (London) 566, 245 (2019).

[23] L. Gross, F. Mohn, N. Moll, P. Liljeroth, and G. Meyer, Science 325, 1110 (2009).

[24] F. Neese, Comput. Mol. Sci. 2, 73 (2012).

[25] J. Repp, G. Meyer, F. E. Olsson, and M. Persson, Science 305, 493 (2004).

[26] See Supplemental Material at http://link.aps.org/ supplemental/10.1103/PhysRevLett.125.176803 for the determination of the charge state and molecular adsorption position, simulated constant-height AC-STM image and experimental line profiles, and further AFM images.

[27] L. Gross, F. Mohn, N. Moll, G. Meyer, R. Ebel, W. M. Abdel-Mageed, and M. Jaspars, Nat. Chem. 2, 821 (2010).

[28] N. Pavliček, B. Fleury, M. Neu, J. Niedenführ, C. Herranz Lancho, M. Ruben, and J. Repp, Phys. Rev. Lett. 108, 086101 (2012).

[29] C.-A. Palma, S. Joshi, T. Hoh, D. Ecija, J. V. Barth, and W. Auwärter, Nano Lett. 15, 2242 (2015).

[30] M. Wagner, J. Hofinger, M. Setvin, L. A. Boatner, M. Schmid, and U. Diebold, ACS Appl. Mater. Interfaces 10, 14175 (2018).

[31] K.-F. Braun and S.-W. Hla, Nano Lett. 5, 73 (2005).

[32] F. Mohn, Dissertation, IBM Zurich and University of Regensburg, 2012.

[33] F. Albrecht, N. Pavliček, C. Herranz-Lancho, M. Ruben, and J. Repp, J. Am. Chem. Soc. 137, 7424 (2015).

[34] F. Albrecht, F. Bischoff, W. Auwärter, J. V. Barth, and J. Repp, Nano Lett. 16, 7703 (2016).

[35] L. L. Patera, Z. Zou, C. Dri, C. Africh, J. Repp, and G. Comelli, Phys. Chem. Chem. Phys. 19, 24605 (2017). 
[36] B. Schuler, W. Liu, A. Tkatchenko, N. Moll, G. Meyer, A. Mistry, D. Fox, and L. Gross, Phys. Rev. Lett. 111, 106103 (2013).

[37] W. Steurer, S. Fatayer, L. Gross, and G. Meyer, Nat. Commun. 6, 8353 (2015).

[38] S. Fatayer, B. Schuler, W. Steurer, I. Scivetti, J. Repp, L. Gross, M. Persson, and G. Meyer, Nat. Nanotechnol. 13, 376 (2018).

[39] P. Scheuerer, L. L. Patera, F. Simbürger, F. Queck, I. Swart, B. Schuler, L. Gross, N. Moll, and J. Repp, Phys. Rev. Lett. 123, 066001 (2019).
[40] L. Gross, F. Mohn, P. Liljeroth, J. Repp, F. J. Giessibl, and G. Meyer, Science 324, 1428 (2009).

[41] T. Koopmans, Physica (Amsterdam) 1, 104 (1934).

[42] P. Scheuerer, L. L. Patera, and J. Repp, Nano Lett. 20, 1839 (2020).

[43] L. L. Patera, F. Queck, P. Scheuerer, N. Moll, and J. Repp, Phys. Rev. Lett. 123, 016001 (2019).

[44] S. Fatayer, F. Albrecht, Y. Zhang, D. Urbonas, D. Peña, N. Moll, and L. Gross, Science 365, 142 (2019). 